\title{
Study of Serum Vitamin D, Calcium and Phosphorus in the Uncomplicated and Complicated cases of Type 2 Diabetes Mellitus
}

\author{
Akanksha Menaria $^{1^{*}}$, Dr. A K Verma ${ }^{2}$, Shilpa Sharma ${ }^{3}$
}

${ }^{1}$ Biochemist, Department of Biochemistry, RNT Medical College, Udaipur, Rajasthan, India

${ }^{2}$ Professor \& Head, Additional Principal, Department of Biochemistry, RNT Medical College, Udaipur, Rajasthan, India

${ }^{3}$ Senior Demonstrator, Department of Biochemistry, RNT Medical College, Udaipur, Rajasthan, India

DOI: $10.36347 /$ sjams.2020.v08i12.005

| Received: 18.11 .2020 | Accepted: 01.12.2020 | Published: 07.12.2020

*Corresponding author: Akanksha Menaria

Abstract

Original Research Article

Background: Diabetes mellitus also described as "Disease of Civilization" is a metabolic anamoly having gruesome impact on quality of life worldwide. The disease has been labeled since ancient times and was recognized as a serious illness. Objectives: The objectives of the study are to estimate the level of Serum Vitamin D, Calcium and Phosphorus in the Uncomplicated and complicated cases of Type 2 Diabetes Melitus. Materials \& Methods: The work encloses clinical study on Type 2 Diabetes Mellitus. It is broadly categorized into two parts. The first part comprises of Control group study conducted on 100 healthy control subjects. The second part comprises of Clinical Study, further subdivided into two groups. The first group (Group 1) comprises of 100 newly diagnosed or Uncomplicated Type 2 Diabetic cases and the second group(Group 2) comprises of 100 Complicated (Microvascular or Macrovascular) Type 2 Diabetic cases. Patients and controls were selected from the outdoor and indoor area of Endocrinology and Medicine Department of M.B. Hospital, R.N.T. Medical College, Udaipur.Subjects of both Group I and II along with control group were analyzed for serum Vitamin D, S.Calcium, S.Phosphorus, FBS, PPBS, $\mathrm{HbA}_{1} \mathrm{C}$, Lipid Profile and Liver Function Test. After assessing all the values, Mean and standard deviation of all subjects and parameters are analyzed. Statistical analysis is performed with SPSS software. Comparison of categorical variables (among category comparison) is done using Chi-Square Test. Comparison between cases and control is done by independent student's $t$ test. By using t value $\mathrm{P}$ value is calculated. $\mathrm{P}$ value less than $0.05(\mathrm{P}<0.05)$ is considered significant. Results: All the categories of Complicated Type 2 Diabetic cases when compared with Control of the same category gave a similar reflection of significantly low Vitamin $\mathrm{D}(\mathrm{P}<0.05)$ Calcium, Phosphorus $(\mathrm{P}<0.05)$, HDL-C, Total Protein, Albumin $(\mathrm{P}<0.05)$ and significantly high glycemic status $(\mathrm{P}<0.00)$, Total Cholesterol, TG, LDL-C, VLDL-C $(\mathrm{P}<0.00)$, Urea, Creatinine, Uric acid, SGOT and Alkaline Phosphatase $(\mathrm{P}<0.00)$. The Smokers displayed significantly lower Vitamin $\mathrm{D}$, and raised FBS, LDL-C, Uric acid $(\mathrm{P}<0.05)$. The Alcoholics exhibited drop in Vitamin $\mathrm{D}, \mathrm{HDL}-\mathrm{C}(\mathrm{P}<0.05)$ and significant upsurge of Total Cholesterol, TG, LDL-C $(\mathrm{P}<0.05)$. Conclusion: Our study clearly indicates significant decline in Vitamin D, Calcium and Phosphorus levels in Type 2 Diabetes and its associated complications. Faulty lifestyle, altered food habits \& diminished physical activity culpable for obesity aggravates the pre-existing insulin resistance state. Therefore timely assessing of Vitamin D and various minerals at the start and even before the onset of diabetes will be certainly supportive in diabetes management. Towering figure of this epidemic demands varied biochemical approach along with conventional glucose monitoring goals. Hence, our study strongly advocates the regular assessment of Vitamin D and Minerals for beforehand diagnosis of the diabetes and its vascular adversities.

Key words: Vitamin D, DM, Minerals, smoking,alocohol.

Copyright $(92020$ The Author(s): This is an open-access article distributed under the terms of the Creative Commons Attribution 4.0 International License (CC BY-NC 4.0) which permits unrestricted use, distribution, and reproduction in any medium for non-commercial use provided the original author and source are credited.

\section{INTRODUCTION}

Type 2 Diabetes also called Adult onset Diabetes or Non-Insulin Dependent Diabetes Mellitus includes a group of different metabolic disorder resulting in an elevated blood glucose level (hyperglycemia) secondary to either insulin deficiency or abnormal insulin action. The symptoms of untreated diabetes include excessive urine production, increased hunger and poor healing [1]. Diabetes is a clinical syndrome characterized by hyperglycemia caused by absolute or relative deficiency of insulin.

Vitamin D has an important role in the regulation of cellular $\mathrm{Ca}^{+2}$ signalling which is linked to cellular responses, signaling and secretion. Sustained $\mathrm{Ca}^{+2}$ signals triggered by $1,25-(\mathrm{OH})_{2 \mathrm{D} 3}$ have been researched for the regulation of apoptosis in diseases as obesity and Type 2 diabetes [2]. Moreover, 1, 25-(OH) 
2D3 induced $\mathrm{Ca}^{+2}$ signals $\left(\mathrm{Ca}^{+2}\right.$ oscillations $)$ can regulate insulin secretion from pancreatic $\beta$-cells [3]. The rapid increase in intracellular calcium triggers insulin release. The role of $1,25(\mathrm{OH}) 2 \mathrm{D} 3$ in insulin secretion derives from its effect on $\mathrm{Ca}+2$ influx, mobilization, and buffering in pancreatic $\beta$-cells [4].

Calcium is essential for insulin mediated extracellular processes in insulin responsive tissues such as skeletal muscle and adipose tissue with a very narrow range of $\mathrm{Ca}+2$ needed for optimal insulin mediated function. Changes in calcium in primary insulin target tissue may contribute to peripheral insulin resistance (21). Low calcium causes impairment of insulin receptor phosphorylation, a calcium-dependent process, leading to impaired insulin signal transduction and decreased glucose transporter-4 activity $[5,6]$. Moreover changes in calcium modulate adipocyte metabolism, which may promote triglyceride accumulation via increased de novo lipogenesis and inability to suppress insulin mediated lipolysis leading to fat accumulation $[7,8]$. Changes in serum $\mathrm{Ca}+2$ may also lead to cykotine induced apoptosis [9].

\section{Material and Methodology}

The work encloses clinical study on Type 2 Diabetes Mellitus. It is broadly categorized into two parts. The first part comprises of Control group study conducted on 100 healthy control subjects. The second part comprises of Clinical Study, further subdivided into two groups. The first group (Group 1) comprises of 100 newly diagnosed or Uncomplicated Type 2 Diabetic cases and the second group (Group 2) comprises of 100 Complicated (Microvascular or Macrovascular) Type 2 Diabetic cases. Patients and controls were selected from the outdoor and indoor area of Endocrinology and Medicine Department of M.B. Hospital, R.N.T. Medical College, Udaipur.

Subjects of both Group I and II along with control group were analyzed for serum Vitamin D, S.Calcium, S.Phosphorus, FBS, PPBS, $\mathrm{HbA}_{1} \mathrm{C}$, Lipid Profile and Liver Function Test.

\section{Exclusion criteria}

Cancer, Renal Osteodystrophy patients ,Patients having anemia of any cause, serious infections, chronic liver disease or on corticosteroid therapy.Patients receiving medications that affect vitamin D metabolism/ absorption (phenytoin, rifampin, isoniazid, ketocanazole).Patients receiving vitamin D and Calcium supplementation

$10 \mathrm{ml}$ of blood from the Control group and Clinical group was drawn from a antecubital vein and collected in plain vial. Serum was separated by centrifugation of blood sample and following parameters were estimated in both Control and Clinical study group.
1) Vitamin D - ECLIA method on Cobas e411 analyzer

2) Calcium - o-crespothalin complexone (OCPC) method on Siemens Dimension $\mathrm{R}_{\mathrm{x}} \mathrm{L}$ Clinical Chemistry System

3) Phosphorus - phosphomolybdate method on Siemens Dimension $\mathrm{R}_{\mathrm{x}} \mathrm{L}$ Clinical Chemistry System

4) Blood sugar - hexokinase-glucose-6 phosphate dehydrogenase method on Siemens Dimension $\mathrm{R}_{\mathrm{x}} \mathrm{L}$ Clinical Chemistry System

5) HbA1C: $\mathrm{HbA}_{1} \mathrm{C}$ values are taken from the patient medical record.

6) Lipid profile

- S. cholesterol: enzymatic method on Siemens Dimension $\mathrm{R}_{\mathrm{x}} \mathrm{L}$ Clinical Chemistry System

- S. Triglyceride: enzymatic method on Siemens Dimension $\mathrm{R}_{\mathrm{x}} \mathrm{L}$ Clinical Chemistry System

- S.HDL: enzymatic method on Siemens Dimension $\mathrm{R}_{\mathrm{x}} \mathrm{L}$ Clinical Chemistry System

- S.LDL: enzymatic method on Siemens Dimension $\mathrm{R}_{\mathrm{x}} \mathrm{L}$ Clinical Chemistry System

- S.VLDL: The value of VLDL-cholesterol is calculated by friedwald's formula. VLDLcholesterol $=$

- $\quad$ Triglyceride/5

7) Liver function test

- SGPT (ALT): IFCC method on Siemens Dimension $\mathrm{R}_{\mathrm{x}} \mathrm{L}$ Clinical Chemistry System

- SGOT (AST): IFCC method on Siemens Dimension $\mathrm{R}_{\mathrm{x}} \mathrm{L}$ Clinical Chemistry System

- ALP: IFCC method on Siemens Dimension $\mathrm{R}_{\mathrm{x}} \mathrm{L}$ Clinical Chemistry System

- Total protein: modified biuret method on Siemens Dimension $\mathrm{R}_{\mathrm{x}} \mathrm{L}$ Clinical Chemistry System

- Albumin: bromocresol purple (BCP) dye-binding method on Siemens Dimension $R_{x} L$ Clinical Chemistry System

BMI, Waist circumference (WC), Waist -Hip ratio (W/HR) is measured as per WHO guidelines (WHO, 2014).

\section{Statistical Analysis}

After assessing all the values, Mean and standard deviation of all subjects and parameters are analyzed. Statistical analysis is performed with SPSS software. Comparison of categorical variables (among category comparison) is done using Chi-Square Test. Comparison between cases and control is done by independent student's $t$ test. By using $t$ value $P$ value is calculated. $\mathrm{P}$ value less than $0.05(\mathrm{P}<0.05)$ is considered significant. Multiple comparisons are done by Post Hoc Analysis of Variance (ANOVA) and Least Significant Difference (LSD) is calculated using Fisher's LSD Method. Using LSD, $t$ and $\mathrm{P}$ values are calculated.

\section{RESUlTS AND DisCUSSION}


Table-1: Age wise distribution of participants

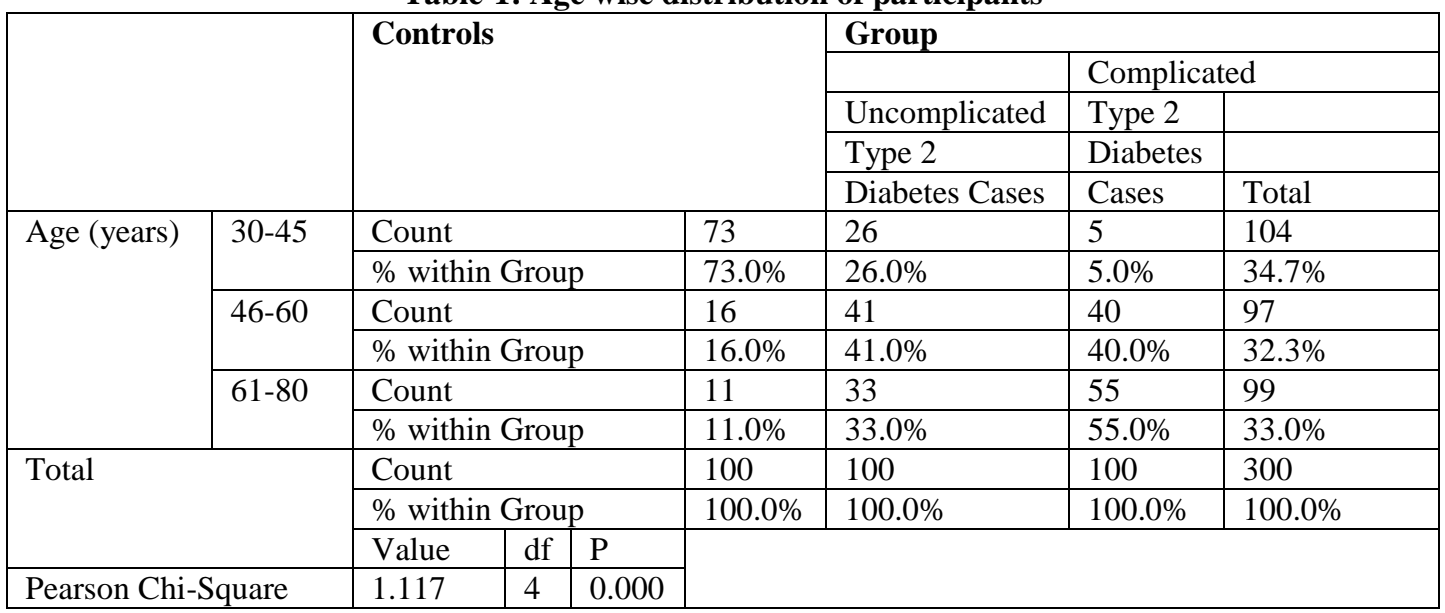

Table 1 presents the comparison of age group which is divided into three sub group; 30-45 years, 4660 years and 61-80 years showed valid percentage of $73.0 \%, 16.0 \%$ and $11.0 \%$ respectively in Controls, $26.0 \%, 41.0 \%$ and $33.0 \%$ respectively in
Uncomplicated Type2 Diabetes Cases and 5.0\%, 40.0\% and $55.0 \%$ respectively in Complicated Type2 Diabetes Cases. The value obtained from Pearson Chi-Square test is 1.117 which is statistically significant $(\mathrm{P}<0.000)$.

Table-2: sex wise distribution of participants

\begin{tabular}{|c|c|c|c|c|c|c|c|c|c|}
\hline & & & & & & Group & & Comp & \\
\hline & & Control & & & & Uncomp & icated & Type 2 & \\
\hline & & & & & & Type 2 I & iabetes & Diabet & \\
\hline & & & & & & Cases & & Cases & Total \\
\hline Sex & Male & Count & & & 64 & 40 & 53 & 157 & \\
\hline & & $\%$ withi & $\mathrm{Gr}$ & & $64.0 \%$ & $40.0 \%$ & $53.0 \%$ & $52.3 \%$ & \\
\hline & Female & Count & & & 36 & 60 & 47 & 143 & \\
\hline & & $\%$ withi & $\mathrm{Gr}$ & & $36.0 \%$ & $60.0 \%$ & $47.0 \%$ & $47.7 \%$ & \\
\hline Total & & Count & & & 100 & 100 & 100 & 300 & \\
\hline & & $\%$ withi & $\mathrm{Gr}$ & & $100.0 \%$ & $100.0 \%$ & $100.0 \%$ & $100.0 \%$ & \\
\hline & & Value & $\mathrm{df}$ & $\mathrm{P}$ & & & & & \\
\hline Pears & Chi-Square & 11.572 & 2 & 0.003 & & & & & \\
\hline
\end{tabular}

Table 2 represents the Sex group (Male \& Female) comparison of Cases and Controls with valid percentage of $64.0 \%$ and $36.0 \%$ respectively in Controls, $40.0 \%$ and $60.0 \%$ respectively in
Uncomplicated Type2 Diabetes Cases and 53.0\% and $47.0 \%$ respectively in Complicated Type2 Diabetes Cases. Chi-Square test yielded a statistically significant value of $11.572(\mathrm{P}<0.003)$.

Table-3: comparison of BMI between case and control

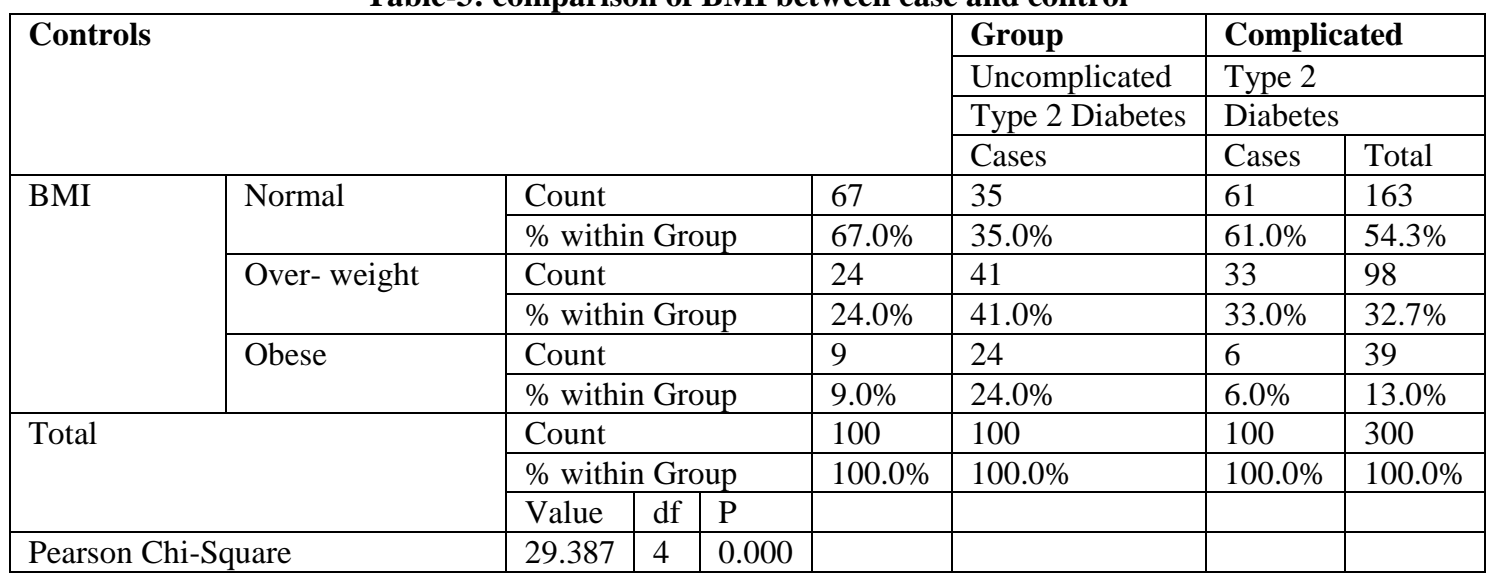

Table 3 represents comparison of BMI groups categorized into Normal, Overweight and Obese with valid percentage of $67.0 \%, 24.0 \%$ and $9.0 \%$ respectively in Controls, 35.0\%, $41.0 \%$ and $24.0 \%$ 
respectively in Uncomplicated Type2 Diabetes Cases and $61.0 \%, 33.0 \%$ and $6.0 \%$ respectively in
Complicated Type2 Diabetes Cases. Chi-Square value obtained is 29.387 with a significant $\mathrm{P}$ value $(\mathrm{P}<0.000)$.

Table-4: Statistical Evaluation of Vitamin D, Mineral Status, Glycemic Status, Lipid Profile and other Biochemical Parameters among Uncomplicated Type 2 Diabetes cases (Intra group) for the Disease Duration (Years)

\begin{tabular}{|c|c|c|c|c|c|c|c|c|c|c|c|c|}
\hline \multirow[t]{2}{*}{ Parameters } & \multicolumn{2}{|c|}{$\begin{array}{l}0-1 \mathrm{Vs} \\
>1-5\end{array}$} & \multicolumn{2}{|c|}{$\begin{array}{lll}0-1 & V s & >5- \\
10 & & \\
\end{array}$} & \multicolumn{2}{|c|}{$0-1 \mathrm{Vs}>10+$} & \multicolumn{2}{|c|}{$\begin{array}{l}>1-5 \mathrm{Vs} \\
>5-10\end{array}$} & \multicolumn{2}{|c|}{$>1-5 \mathrm{Vs}>10+$} & \multicolumn{2}{|c|}{$\begin{array}{l}>5-10 \mathrm{Vs} \\
>10+\end{array}$} \\
\hline & t & signi & t & signi & $\mathbf{t}$ & signi & t & signi & t & signi & $\mathbf{t}$ & signi \\
\hline $\begin{array}{l}\text { Vitamin D } \\
(\mathrm{ng} / \mathrm{ml})\end{array}$ & 2.20 & 0.05 & 1.99 & 0.05 & 2.33 & 0.05 & 5.20 & 0.05 & 2.88 & 0.05 & 1.89 & 0.05 \\
\hline Calcium (mg/dl) & 2.56 & 0.05 & 3.23 & 0.05 & 5.12 & 0.01 & 2.32 & 0.05 & 2.43 & 0.05 & 1.11 & N.S \\
\hline $\begin{array}{l}\text { Phosphorus } \\
(\mathbf{m g} / \mathbf{d l})\end{array}$ & 0.12 & N.S & 0.18 & N.S & 0.16 & N.S & 0.06 & N.S & 0.04 & N.S & 0.02 & N.S \\
\hline FBS (mg/dl) & 0.81 & N.S & 5.15 & 0.01 & 4.73 & 0.05 & 2.33 & 0.05 & 1.91 & 0.05 & 0.41 & N.S \\
\hline PPBS (mg/dl) & 2.20 & 0.05 & 6.94 & 0.01 & 9.32 & 0.001 & 6.74 & 0.01 & 8.12 & 0.005 & 6.38 & 0.01 \\
\hline $\mathbf{H b A}_{1} \mathbf{C}(\%)$ & 0.14 & N.S & 0.60 & N.S & 1.95 & 0.001 & 0.45 & N.S & 1.89 & 0.05 & 1.88 & 0.05 \\
\hline $\begin{array}{l}\text { T.Cholesterol } \\
\text { (mg/dl) }\end{array}$ & 0.29 & N.S & 2.47 & 0.05 & $\begin{array}{l}16.7 \\
5 \\
\end{array}$ & 0.000 & 1.18 & N.S & $\begin{array}{l}16.4 \\
6\end{array}$ & 0.000 & 14.28 & 0.000 \\
\hline $\begin{array}{l}\text { Triglycerides } \\
(\mathrm{mg} / \mathrm{dl})\end{array}$ & 0.71 & N.S & 1.33 & N.S & 2.40 & 0.05 & 0.61 & N.S & 2.33 & 0.05 & 2.73 & 0.05 \\
\hline HDL-C (mg/dl) & 0.80 & N.S & 2.55 & 0.05 & 3.40 & 0.05 & 0.25 & N.S & 3.20 & 0.05 & 0.95 & N.S \\
\hline LDL-C (mg/dl) & 2.83 & 0.05 & 3.96 & 0.05 & 7.80 & 0.01 & 1.79 & 0.05 & 6.96 & 0.01 & 5.76 & 0.01 \\
\hline VLDL-C (mg/dl) & 0.14 & N.S & 1.26 & N.S & 2.68 & 0.05 & 1.12 & N.S & 2.82 & N.S & 2.94 & 0.05 \\
\hline SGOT (U/L) & 0.57 & N.S & 0.45 & N.S & 0.97 & N.S & 1.02 & N.S & 1.40 & N.S & 1.43 & N.S \\
\hline SGPT (U/L) & 0.64 & N.S & 1.38 & N.S & 1.24 & N.S & 1.73 & N.S & 0.88 & N.S & 0.62 & N.S \\
\hline $\begin{array}{l}\text { Alk.Phosphatase } \\
\text { (U/L) }\end{array}$ & 0.67 & N.S & 2.45 & 0.04 & 1.51 & N.S & 1.77 & N.S & 0.84 & N.S & 0.21 & N.S \\
\hline $\begin{array}{l}\text { Total Protein } \\
\text { (g/dl) }\end{array}$ & 1.35 & N.S & 2.15 & 0.05 & 2.47 & 0.05 & 3.51 & 0.05 & 4.11 & 0.05 & 0.62 & N.S \\
\hline Albumin (g/dl) & 0.11 & N.S & 2.07 & 0.05 & 2.42 & 0.05 & 0.84 & N.S & 1.88 & 0.05 & 0.01 & N.S \\
\hline
\end{tabular}

Table 4 represents the comparison of Duration of Disease divided into 0-1year, >1-5Years, >5-10years and $>10+$ years with valid percentage of $34.0 \%, 49.0 \%$, $12.0 \%$ and $5.0 \%$ respectively in Uncomplicated Type 2
Diabetes Cases and $13.0 \%, 35.0 \%, 26.0 \%$ and $26.0 \%$ respectively in Complicated Type2 Diabetes Cases. Chi- square value obtained in total 200 subjects is 3.467 and a statistically significant $\mathrm{P}$ value $(\mathrm{P}<0.000)$.

Table-5: Statistical Evaluation of Vitamin D, Mineral Status, Glycemic Status, Lipid Profile and other Biochemical Parameters among Complicated Type 2 Diabetes cases (Intra group) for the Disease Duration (Years)

\begin{tabular}{|c|c|c|c|c|c|c|c|c|c|c|c|c|}
\hline \multirow[b]{2}{*}{ Parameters } & \multicolumn{2}{|c|}{$0-1$ Vs $>1-5$} & \multicolumn{2}{|c|}{$0-1 \mathrm{Vs}>5-10$} & \multicolumn{2}{|c|}{$0-1 \mathrm{Vs}>10+$} & \multicolumn{2}{|c|}{$\begin{array}{c}>1-5 \mathrm{Vs} \\
>5-10\end{array}$} & \multicolumn{2}{|c|}{$\begin{array}{c}>1-5 \mathrm{Vs} \\
>10+\end{array}$} & \multicolumn{2}{|c|}{$\begin{array}{c}>5-10 \mathrm{Vs} \\
>10+\end{array}$} \\
\hline & $\mathbf{t}$ & signi & $\mathbf{t}$ & signi & $\mathbf{t}$ & signi & $\mathbf{t}$ & signi & $\mathbf{t}$ & signi & $\mathbf{t}$ & signi \\
\hline Vitamin D (ng/ml) & 1.91 & 0.05 & 0.21 & N.S & 4.74 & 0.05 & 0.39 & N.S & 3.11 & 0.05 & 3.84 & 0.05 \\
\hline Calcium (mg/dl) & 0.71 & N.S & 2.77 & 0.05 & 3.01 & 0.05 & 0.69 & N.S & 0.58 & N.S & 0.08 & N.S \\
\hline Phosphorus (mg/dl) & 1.60 & N.S & 1.91 & 0.05 & 3.28 & 0.05 & 0.84 & N.S & 2.11 & 0.05 & 1.97 & 0.05 \\
\hline FBS (mg/dl) & 0.29 & N.S & 1.00 & N.S & 3.21 & 0.05 & 0.37 & N.S & 2.89 & 0.05 & 1.12 & N.S \\
\hline PPBS (mg/dl) & 0.56 & N.S & 1.21 & N.S & 2.78 & 0.05 & 0.39 & N.S & 2.52 & 0.05 & 2.11 & 0.05 \\
\hline $\mathrm{HbA}_{1} \mathrm{C}(\%)$ & 0.43 & N.S & 0.84 & N.S & 2.43 & 0.05 & 0.61 & N.S & 1.99 & 0.05 & 0.32 & N.S \\
\hline T.Cholesterol (mg/dl) & 0.11 & N.S & 0.62 & N.S & 0.58 & N.S & 0.33 & N.S & 0.21 & N.S & 0.14 & N.S \\
\hline Triglycerides (mg/dl) & 0.96 & N.S & 0.84 & N.S & 1.80 & N.S & 0.21 & N.S & 0.32 & N.S & 0.27 & N.S \\
\hline HDL-C (mg/dl) & 0.19 & N.S & 0.20 & N.S & 0.22 & N.S & 0.10 & N.S & 0.21 & N.S & 0.14 & N.S \\
\hline LDL-C (mg/dl) & 0.06 & N.S & 0.37 & N.S & 0.04 & N.S & 0.01 & N.S & 0.29 & N.S & 0.31 & N.S \\
\hline VLDL-C (mg/dl) & 0.96 & N.S & 0.89 & N.S & 1.62 & N.S & 0.12 & N.S & 0.10 & N.S & 0.84 & N.S \\
\hline SGOT (U/L) & 0.72 & N.S & 0.84 & N.S & 0.62 & N.S & 0.51 & N.S & 0.18 & N.S & 0.39 & N.S \\
\hline SGPT (U/L) & 0.45 & N.S & 2.45 & 0.05 & 0.12 & N.S & 0.46 & N.S & 0.78 & N.S & 1.89 & 0.05 \\
\hline Alk.Phosphatase (U/L) & 0.47 & N.S & 0.04 & N.S & 0.44 & N.S & 0.46 & N.S & 0.05 & N.S & 0.39 & N.S \\
\hline Total Protein $(\mathrm{g} / \mathrm{dl})$ & 0.86 & N.S & 2.11 & 0.05 & 2.57 & 0.05 & 0.99 & N.S & 2.19 & 0.05 & 0.81 & N.S \\
\hline Albumin (g/dl) & 1.68 & N.S & 1.17 & N.S & 1.10 & N.S & 0.21 & N.S & 0.07 & N.S & 0.01 & N.S \\
\hline
\end{tabular}


Table-6: Macrovascular Complications * Group Cases and Controls (CVD: cardiovascular disease, HT: Hypertension)

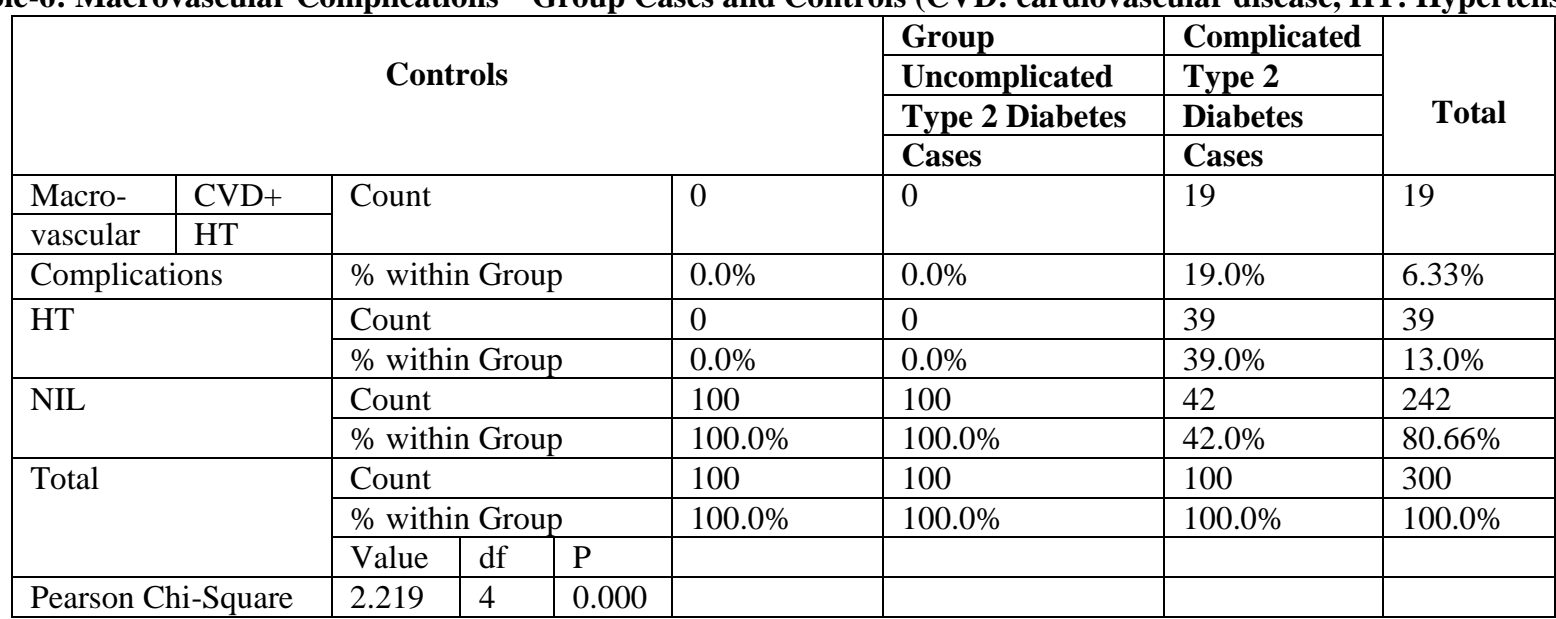

Table-7: Microvascular Complications * Group Cases and Controls (NRP:neuropathy. RP:Retinopathy ,NHP:Nephropathy)

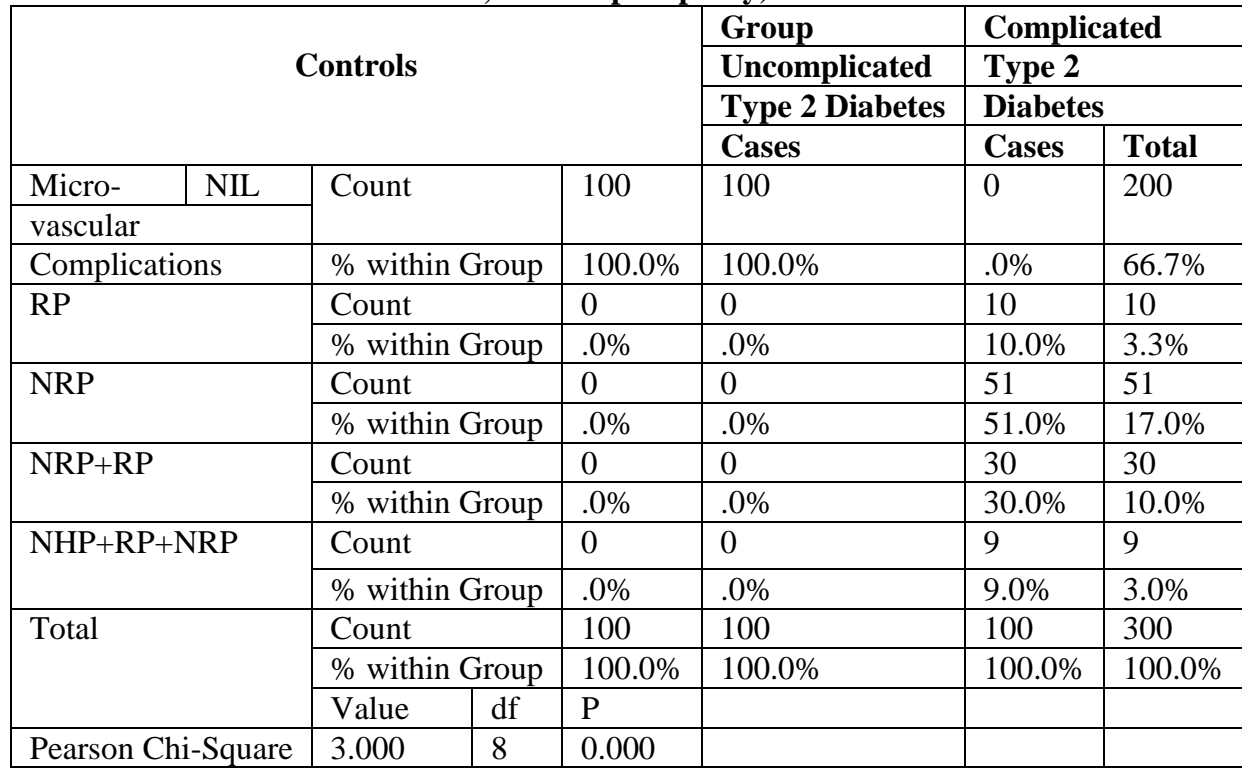

Table-8: Statistical Evaluation of Vitamin D, Glycemic Status, Lipid Profile and other Biochemical Parameters among Control Vs Uncomplicated Type 2 Diabetes cases for Gender and Inhabitance

\begin{tabular}{|c|c|c|c|c|c|c|c|c|c|c|}
\hline \multirow[t]{3}{*}{ Parameters } & \multirow{2}{*}{\multicolumn{3}{|c|}{ Total }} & \multicolumn{4}{|c|}{ Gender } & \multicolumn{3}{|c|}{ Inhabitance } \\
\hline & & & & \multicolumn{2}{|c|}{ Male } & \multicolumn{2}{|c|}{ Female } & \multicolumn{2}{|c|}{ Urban } & \multirow{2}{*}{$\begin{array}{l}\text { Rural } \\
\text { signi }\end{array}$} \\
\hline & $\mathbf{t}$ & signi & $\mathbf{t}$ & signi & $\mathbf{t}$ & signi & t & signi & $\mathbf{t}$ & \\
\hline Vitamin D (ng/ml) & 2.10 & 0.05 & 3.14 & 0.05 & 2.60 & 0.05 & 3.05 & 0.05 & 3.20 & 0.05 \\
\hline Phosphorus (mg/dl) & 1.13 & N.S & 0.904 & N.S & 1.22 & N.S & 1.83 & N.S & 0.72 & N.S \\
\hline FBS (mg/dl) & 41.74 & 0.00 & 25.24 & 0.000 & 16.90 & 0.000 & 10.15 & 0.000 & 31.15 & 0.000 \\
\hline PPBS (mg/dl) & 70.15 & 0.00 & 36.36 & 0.000 & 31.71 & 0.000 & 14.81 & 0.000 & 54.88 & 0.000 \\
\hline $\mathrm{HbA}_{1} \mathrm{C}(\%)$ & 163.61 & 0.00 & 110.67 & 0.000 & 53.43 & 0.000 & 38.15 & 0.000 & 122.43 & 0.000 \\
\hline T.Cholesterol (mg/dl) & 2.59 & 0.50 & 0.719 & N.S & 1.15 & N.S & 0.30 & N.S & 5.86 & 0.005 \\
\hline Triglycerides (mg/dl) & 4.31 & 0.01 & 0.501 & N.S & 11.92 & 0.000 & 0.89 & N.S & 3.15 & 0.05 \\
\hline HDL-C (mg/dl) & 2.22 & 0.50 & 0.06 & N.S & 1.65 & N.S & 1.89 & N.S & 0.40 & N.S \\
\hline LDL-C (mg/dl) & 3.45 & 0.05 & 3.35 & 0.05 & 7.57 & 0.001 & 1.74 & N.S & 1.88 & N.S \\
\hline VLDL-C (mg/dl) & 4.31 & 0.01 & 0.50 & N.S & 11.92 & 0.000 & 0.89 & N.S & 3.15 & 0.05 \\
\hline SGOT (U/L) & 5.64 & 0.005 & 1.00 & N.S & 7.54 & 0.05 & 2.97 & 0.056 & 2.52 & N.S \\
\hline SGPT (U/L) & 0.93 & N.S & 0.39 & N.S & 3.72 & 0.05 & 1.44 & N.S & 0.08 & N.S \\
\hline Alk.Phosphatase (U/L) & 11.73 & 0.000 & 3.95 & 0.05 & 9.39 & 0.000 & 1.27 & N.S & 1.30 & N.S \\
\hline Total Protein $(\mathrm{g} / \mathrm{dl})$ & 0.76 & N.S & 0.46 & N.S & 1.50 & N.S & 0.72 & N.S & 0.90 & N.S \\
\hline Albumin (g/dl) & 1.07 & N.S & 1.83 & N.S & 0.361 & N.S & 1.71 & N.S & 0.62 & N.S \\
\hline
\end{tabular}


Table-9: Statistical Evaluation of Vitamin D, Mineral Status, Glycemic Status, Lipid Profile and other Biochemical Parameters among Uncomplicated Type 2 Diabetes cases (Intra group) for Educational Status, Family History (FH) and Dietary Habits

\begin{tabular}{|c|c|c|c|c|c|c|c|c|c|c|}
\hline \multirow[t]{3}{*}{ Parameters } & \multirow{2}{*}{\multicolumn{2}{|c|}{$\begin{array}{l}\text { Illiterate Vs } \\
\text { Semi } \\
\text { Literate }\end{array}$}} & \multirow{2}{*}{\multicolumn{2}{|c|}{$\begin{array}{l}\text { Illiterate Vs } \\
\text { Literate }\end{array}$}} & \multirow{2}{*}{\multicolumn{2}{|c|}{$\begin{array}{l}\text { Semi-Literate } \\
\text { Vs Literate }\end{array}$}} & \multirow{2}{*}{\multicolumn{2}{|c|}{$\begin{array}{l}\text { With FH Vs } \\
\text { without FH }\end{array}$}} & \multirow{2}{*}{\multicolumn{2}{|c|}{$\begin{array}{l}\text { Vegetarian } \\
\text { Vs Non } \\
\text { Vegetarian } \\
\end{array}$}} \\
\hline & & & & & & & & & & \\
\hline & $\mathbf{t}$ & signi & t & signi & t & signi & t & signi & $\mathbf{t}$ & signi \\
\hline Vitamin D (ng/ml) & 1.53 & N.S & 0.34 & N.S & 0.53 & N.S & 1.10 & N.S & 0.88 & N.S \\
\hline Zinc ( $\mu \mathrm{g} / \mathrm{dl})$ & 0.98 & N.S & 5.85 & 0.005 & 3.24 & 0.005 & 0.67 & N.S & 0.57 & N.S \\
\hline Magnesium (mg/dl) & 0.81 & N.S & 1.03 & N.S & 1.17 & N.S & 0.87 & N.S & 0.85 & N.S \\
\hline Calcium (mg/dl) & 0.84 & N.S & 0.54 & N.S & 1.16 & N.S & 1.16 & N.S & 0.89 & N.S \\
\hline Phosphorus (mg/dl) & 1.17 & N.S & 0.22 & N.S & 0.02 & N.S & 0.03 & N.S & 0.33 & N.S \\
\hline FBS (mg/dl) & 2.53 & 0.05 & 8.56 & 0.00 & 1.16 & N.S & 0.13 & N.S & 0.31 & N.S \\
\hline PPBS (mg/dl) & 1.05 & N.S & 7.67 & 0.00 & 6.37 & 0.01 & 0.71 & N.S & 0.35 & N.S \\
\hline $\mathrm{HbA}_{1} \mathbf{C}(\%)$ & 0.75 & N.S & 1.37 & N.S & 0.28 & N.S & 0.88 & N.S & 0.11 & N.S \\
\hline T.Cholesterol (mg/dl) & 1.93 & 0.05 & 2.00 & 0.05 & 0.54 & N.S & 0.46 & N.S & 1.23 & N.S \\
\hline Triglycerides (mg/dl) & 0.83 & N.S & 1.30 & N.S & 2.37 & 0.05 & 1.49 & N.S & 1.15 & N.S \\
\hline HDL-C (mg/dl) & 0.76 & N.S & 3.40 & 0.05 & 1.53 & N.S & 0.34 & N.S & 0.19 & N.S \\
\hline LDL-C (mg/dl) & 1.19 & N.S & 1.44 & N.S & 1.02 & N.S & 2.14 & 0.05 & 0.80 & N.S \\
\hline VLDL-C (mg/dl) & 0.83 & N.S & 0.26 & N.S & 2.47 & 0.05 & 1.49 & N.S & 1.15 & N.S \\
\hline Urea $(\mathrm{mg} / \mathrm{dl})$ & 1.46 & N.S & 1.86 & N.S & 1.22 & N.S & 1.20 & N.S & 0.03 & N.S \\
\hline Creatinine (mg/dl) & 0.82 & N.S & 0.01 & N.S & 1.26 & N.S & 0.87 & N.S & 1.15 & N.S \\
\hline Uric acid(mg/dl) & 2.18 & 0.05 & 2.34 & 0.05 & 0.29 & N.S & 1.10 & N.S & 2.68 & 0.05 \\
\hline SGOT (U/L) & 0.99 & N.S & 1.48 & N.S & 1.81 & N.S & 0.85 & N.S & 1.50 & N.S \\
\hline SGPT (U/L) & 0.15 & N.S & 1.23 & N.S & 1.55 & N.S & 0.35 & N.S & 1.30 & N.S \\
\hline Alk.Phosphatase (U/L) & 0.73 & N.S & 0.65 & N.S & 0.94 & N.S & 1.96 & 0.05 & 0.90 & N.S \\
\hline Total Protein (g/dl) & 1.02 & N.S & 0.69 & N.S & 0.21 & N.S & 0.86 & N.S & 1.27 & N.S \\
\hline Albumin (g/dl) & 1.68 & N.S & 0.85 & N.S & 0.16 & N.S & 0.99 & N.S & 1.98 & 0.05 \\
\hline
\end{tabular}

Table-10: Statistical Evaluation of Vitamin D, Mineral Status, Glycemic Status, Lipid Profile and other Biochemical Parameters among Control Vs Uncomplicated Type 2 Diabetes cases for Smoking and Alcohol Habits

\begin{tabular}{|c|c|c|c|c|c|c|c|c|}
\hline \multirow[t]{3}{*}{ Parameters } & \multicolumn{4}{|c|}{ Smokers } & \multicolumn{3}{|c|}{ Alcoholic } & \multirow[b]{3}{*}{ signi } \\
\hline & \multicolumn{2}{|c|}{ Yes } & \multicolumn{2}{|l|}{ No } & \multicolumn{2}{|c|}{ Yes } & \multirow{2}{*}{$\begin{array}{l}\text { No } \\
\mathbf{t}\end{array}$} & \\
\hline & $\mathbf{t}$ & signi & $\mathbf{t}$ & signi & $\mathbf{t}$ & signi & & \\
\hline Vitamin D (ng/ml) & 1.38 & N.S & 1.97 & 0.05 & 1.93 & 0.05 & 2.07 & 0.05 \\
\hline Calcium (mg/dl) & 2.84 & 0.05 & 0.88 & N.S & 5.37 & 0.001 & 0.90 & N.S \\
\hline Phosphorus (mg/dl) & 1.09 & N.S & 0.68 & N.S & 0.65 & N.S & 1.08 & N.S \\
\hline FBS (mg/dl) & 7.07 & 0.001 & 36.32 & 0.000 & 21.55 & 0.000 & 34.57 & 0.000 \\
\hline PPBS (mg/dl) & 8.03 & 0.001 & 63.43 & 0.000 & 19.42 & 0.000 & 62.57 & 0.000 \\
\hline $\mathrm{HbA}_{1} \mathrm{C}(\%)$ & 26.42 & 0.001 & 137.68 & 0.000 & 37.28 & 0.000 & 143.02 & 0.000 \\
\hline T.Cholesterol (mg/dl) & 2.15 & 0.05 & 1.73 & N.S & 3.23 & 0.05 & 5.45 & 0.01 \\
\hline Triglycerides (mg/dl) & 1.93 & 0.05 & 2.87 & 0.05 & 3.43 & 0.05 & 4.65 & 0.01 \\
\hline HDL-C (mg/dl) & 1.94 & 0.05 & 1.07 & N.S & 2.14 & 0.05 & 1.41 & N.S \\
\hline LDL-C (mg/dl) & 2.78 & 0.05 & 3.74 & 0.01 & 1.31 & N.S & 1.04 & N.S \\
\hline VLDL-C (mg/dl) & 1.93 & 0.05 & 2.87 & 0.05 & 3.43 & 0.05 & 4.65 & 0.01 \\
\hline SGOT (U/L) & 1.03 & N.S & 4.70 & 0.010 & 1.50 & N.S & 1.29 & N.S \\
\hline SGPT (U/L) & 0.57 & N.S & 0.56 & N.S & 0.11 & N.S & 0.71 & N.S \\
\hline Alk.Phosphatase (U/L) & 3.07 & 0.05 & 16.90 & 0.000 & 1.93 & 0.05 & 2.29 & 0.000 \\
\hline Total Protein (g/dl) & 1.97 & 0.05 & 1.14 & N.S & 1.74 & N.S & 0.71 & N.S \\
\hline Albumin (g/dl) & 1.00 & N.S & 0.93 & N.S & 2.40 & 0.05 & 0.80 & N.S \\
\hline
\end{tabular}

In Smokers Vs Non Smokers collation, the Smokers showed lower values for Vitamin D $(24.20 \pm 7.30$ Vs $20.96 \pm 5.70 ; \quad \mathrm{P}<0.05)$ and higher significance for TG $(119.3 \pm 30.3$ Vs $103.2 \pm 19.3$; $\mathrm{P}<0.01)$ and VLDL-c $(23.86 \pm 8.06$ Vs $20.6 \pm 3.80$; $\mathrm{P}<0.01)$. No more significant parameter was found in this group. In Alcoholic Vs Non-alcoholic comparison, the latter showed significantly high values for Vitamin D \& Zinc $(24.58 \pm 8.20$ Vs 20.34 $\pm 7.90 ; \quad \mathrm{P}<0.05$ and $87.68 \pm 9.58$ Vs $80.37 \pm 8.90 ; \mathrm{P}<0.05$ respectively). The Alcoholics showed higher Alkaline Phosphate level $(82.73 \pm 10.3$ Vs $74.63 \pm 8.8 ; \mathrm{P}<0.05)$ when compared to Non-alcoholics. No other significant variable was found in this comparison [Table $10 \& 13]$. 
Akanksha Menaria et al; Sch J App Med Sci, Dec, 2020; 8(12): 2703-2711

Table-11: Statistical Evaluation of Vitamin D, Mineral Status, Glycemic Status, Lipid Profile and other Biochemical Parameters among Complicated Type 2 Diabetes cases (Intra group) for Gender, Inhabitance and Religion

\begin{tabular}{|c|c|c|c|c|c|c|c|c|c|c|}
\hline \multirow{3}{*}{$\begin{array}{l}\text { Parameters } \\
\text { Vitamin D (ng/ml) }\end{array}$} & \multicolumn{2}{|c|}{$\begin{array}{l}\text { Male Vs } \\
\text { Female }\end{array}$} & \multicolumn{2}{|c|}{$\begin{array}{l}\text { Urban Vs } \\
\text { Rural }\end{array}$} & \multicolumn{2}{|c|}{$\begin{array}{l}\text { Hindu Vs } \\
\text { Muslim }\end{array}$} & \multirow{2}{*}{\multicolumn{2}{|c|}{$\begin{array}{l}\text { Hindu Vs } \\
\text { Christian } \\
\text { tsigni }\end{array}$}} & \multirow{2}{*}{\multicolumn{2}{|c|}{$\begin{array}{l}\text { Muslim Vs } \\
\text { Christian } \\
\text { tsigni }\end{array}$}} \\
\hline & $\mathbf{t}$ & signi & $\mathbf{t}$ & signi & $\mathbf{t}$ & signi & & & & \\
\hline & 0.61 & N.S & 3.03 & 0.05 & 3.26 & 0.05 & 1.16 & N.S & 4.84 & 0.05 \\
\hline Calcium (mg/dl) & 2.81 & 0.05 & 0.04 & N.S & 0.23 & N.S & 1.94 & 0.05 & 1.90 & 0.05 \\
\hline Phosphorus (mg/dl) & 0.95 & N.S & 1.13 & N.S & 0.20 & N.S & 1.98 & 0.05 & 1.99 & 0.05 \\
\hline FBS (mg/dl) & 0.54 & N.S & 1.48 & N.S & 4.78 & 0.05 & 0.72 & N.S & 4.74 & 0.05 \\
\hline PPBS (mg/dl) & 0.35 & N.S & 1.57 & N.S & 3.54 & 0.05 & 0.61 & N.S & 1.01 & N.S \\
\hline $\mathrm{HbA}_{1} \mathrm{C}(\%)$ & 0.42 & N.S & 1.74 & N.S & 0.31 & N.S & 0.54 & N.S & 0.36 & N.S \\
\hline T.Cholesterol (mg/dl) & 1.93 & 0.05 & 0.45 & N.S & 1.40 & N.S & 0.29 & N.S & 2.10 & 0.05 \\
\hline Triglycerides (mg/dl) & 1.96 & 0.05 & 0.18 & N.S & 0.12 & N.S & 1.00 & N.S & 0.94 & N.S \\
\hline HDL-C (mg/dl) & 2.10 & 0.05 & 2.50 & 0.010 & 0.09 & N.S & 0.74 & N.S & 0.81 & N.S \\
\hline LDL-C (mg/dl) & 3.00 & 0.05 & 0.25 & N.S & 2.29 & 0.05 & 1.91 & 0.05 & 2.67 & 0.05 \\
\hline VLDL-C (mg/dl) & 1.96 & 0.05 & 0.18 & N.S & 0.12 & N.S & 0.78 & N.S & 0.78 & N.S \\
\hline SGOT (U/L) & 0.73 & N.S & 2.04 & 0.05 & 0.62 & N.S & 2.11 & 0.05 & 1.01 & N.S \\
\hline SGPT (U/L) & 0.35 & N.S & 1.94 & 0.05 & 1.21 & N.S & 2.78 & 0.05 & 1.22 & N.S \\
\hline Alk.Phosphatase (U/L) & 0.58 & N.S & 1.14 & N.S & 1.36 & N.S & 9.67 & 0.01 & 8.69 & 0.01 \\
\hline Total Protein $(\mathrm{g} / \mathrm{dl})$ & 1.06 & N.S & 1.11 & N.S & 0.14 & N.S & 0.74 & N.S & 0.56 & N.S \\
\hline Albumin (g/dl) & 1.43 & N.S & 0.99 & N.S & 0.09 & N.S & 0.31 & N.S & 0.22 & N.S \\
\hline
\end{tabular}

Table-12: Statistical Evaluation of Vitamin D, Mineral Status, Glycemic Status, Lipid Profile and other Biochemical Parameters among Complicated Type 2 Diabetes cases (Intra group) for Educational Status, Family History (FH) and

\begin{tabular}{|c|c|c|c|c|c|c|c|c|c|c|}
\hline \multirow[t]{2}{*}{ Parameters } & \multicolumn{2}{|c|}{$\begin{array}{l}\text { Illiterate Vs } \\
\text { Semi Literate }\end{array}$} & \multicolumn{2}{|c|}{$\begin{array}{l}\text { Illiterate Vs } \\
\text { Literate }\end{array}$} & \multicolumn{2}{|c|}{$\begin{array}{l}\text { Semi-Literate Vs } \\
\text { Literate }\end{array}$} & \multicolumn{2}{|c|}{$\begin{array}{l}\text { With FH Vs } \\
\text { without FH }\end{array}$} & \multicolumn{2}{|c|}{$\begin{array}{l}\text { Vegetarian } \\
\text { Vs Non } \\
\text { Vegetarian }\end{array}$} \\
\hline & $\mathbf{t}$ & signi & $\mathbf{t}$ & signi & $\mathbf{t}$ & signi & $\mathbf{t}$ & signi & t & signi \\
\hline Vitamin D (ng/ml) & 2.81 & 0.05 & 2.96 & 0.05 & 0.18 & N.S & 2.15 & 0.05 & 1.88 & 0.05 \\
\hline Calcium (mg/dl) & 1.04 & N.S & 0.86 & N.S & 0.21 & N.S & 0.74 & N.S & 0.70 & N.S \\
\hline Phosphorus (mg/dl) & 0.43 & N.S & 0.88 & N.S & 0.28 & N.S & 1.88 & 0.05 & 1.29 & N.S \\
\hline FBS (mg/dl) & 0.54 & N.S & 3.11 & 0.05 & 0.86 & N.S & 0.62 & N.S & 0.18 & N.S \\
\hline PPBS (mg/dl) & 0.57 & N.S & 2.66 & 0.05 & 1.24 & N.S & 0.44 & N.S & 0.92 & N.S \\
\hline $\mathrm{HbA}_{1} \mathrm{C}(\%)$ & 0.27 & N.S & 0.31 & N.S & 0.46 & N.S & 0.51 & N.S & 0.57 & N.S \\
\hline T.Cholesterol (mg/dl) & 0.12 & N.S & 0.31 & N.S & 0.58 & N.S & 1.02 & N.S & 1.37 & N.S \\
\hline Triglycerides (mg/dl) & 1.24 & N.S & 1.99 & 0.05 & 1.34 & N.S & 1.00 & N.S & 1.11 & N.S \\
\hline HDL-C (mg/dl) & 0.74 & N.S & 0.89 & N.S & 0.78 & N.S & 1.91 & 0.05 & 1.50 & N.S \\
\hline LDL-C (mg/dl) & 0.29 & N.S & 0.36 & N.S & 0.11 & N.S & 1.21 & N.S & 0.73 & N.S \\
\hline VLDL-C (mg/dl) & 0.24 & N.S & 1.89 & 0.05 & 1.22 & N.S & 1.00 & N.S & 1.11 & N.S \\
\hline SGOT (U/L) & 2.56 & 0.05 & 0.78 & N.S & 0.64 & N.S & 0.17 & N.S & 0.95 & N.S \\
\hline SGPT (U/L) & 1.88 & 0.05 & 0.67 & N.S & 0.50 & N.S & 0.22 & N.S & 0.37 & N.S \\
\hline Alk.Phosphatase (U/L) & 0.22 & N.S & 0.31 & N.S & 0.66 & N.S & 0.91 & N.S & 0.47 & N.S \\
\hline Total Protein $(\mathrm{g} / \mathrm{dl})$ & 0.90 & N.S & 0.91 & N.S & 0.31 & N.S & 1.48 & N.S & 1.13 & N.S \\
\hline Albumin $(\mathrm{g} / \mathrm{dl})$ & 1.91 & 0.05 & 1.89 & 0.05 & 0.51 & N.S & 0.12 & N.S & 0.00 & N.S \\
\hline
\end{tabular}

On the basis of Educational Status, the Cases \& Controls were categorized into Illiterate, SemiLiterate and Literate group. The inter group comparison of Illiterate group gave out significantly low values of Vitamin D $\quad(24.82 \pm 7.01 \quad$ Vs $20.53 \pm 4.97 ; \quad \mathrm{P}<0.05$ significantly high values for $\mathrm{FBS}$, PPBS, $\mathrm{HbA}_{1} \mathrm{C}$ $(\mathrm{P}<0.000)$, Total Cholesterol $\quad(\mathrm{P}<0.01), \quad$ LDL-C $(\mathrm{P}<0.000))$ for the Cases group. For the Semi-Literate group comparison significant low values was recorded for Vitamin D $(24.79 \pm 10.97$ Vs $22.41 \pm 6.34 ; \mathrm{P}<0.05)$ and significant high values for $\mathrm{FBS}$, PPBS, $\mathrm{HbA}_{1} \mathrm{C}$ $(\mathrm{P}<0.000)$, Total Cholesterol, TG, LDL-C, VLDL-C $(\mathrm{P}<0.05)$, Urea $(\mathrm{P}<0.000)$ and Alkaline Phosphatase $(\mathrm{P}<0.05)$ by the Cases group. Comparison of Literate Type 2 Cases Vs Literate Controls reflected significant high values for FBS, PPBS, $\mathrm{HbA}_{1} \mathrm{C}(\mathrm{P}<0.000)$, Total Cholesterol, LDL-C $(\mathrm{P}<0.01)$ and Urea $(\mathrm{P}<0.000)$ by the Cases group [Table 12]. 
Table-13: Statistical Evaluation of Vitamin D, Mineral Status, Glycemic Status, Lipid Profile and other Biochemical Parameters among Control Vs Complicated Type 2 Diabetes cases for Smoking and Alcohol Habits

\begin{tabular}{|c|c|c|c|c|c|c|c|c|}
\hline \multirow[t]{3}{*}{ Parameters } & \multicolumn{5}{|c|}{ Smokers } & \multirow{2}{*}{\multicolumn{3}{|c|}{$\begin{array}{l}\text { Alcoholic } \\
\text { No }\end{array}$}} \\
\hline & \multicolumn{3}{|c|}{\begin{tabular}{|l|l} 
Yes & No \\
\end{tabular}} & \multicolumn{2}{|l|}{ Yes } & & & \\
\hline & $\mathbf{t}$ & signi & $\mathbf{t}$ & signi & $\mathbf{t}$ & signi & $\mathbf{t}$ & signi \\
\hline Vitamin D (ng/ml) & 3.38 & 0.05 & 3.97 & 0.05 & 2.33 & 0.05 & 2.07 & 0.05 \\
\hline Calcium (mg/dl) & 8.84 & 0.001 & 2.88 & 0.05 & 7.37 & 0.001 & 1.90 & 0.05 \\
\hline Phosphorus (mg/dl) & 1.09 & N.S & 0.68 & N.S & 2.65 & 0.05 & 2.08 & 0.04 \\
\hline FBS (mg/dl) & 7.07 & 0.001 & 36.32 & 0.000 & 21.55 & 0.000 & 34.57 & 0.000 \\
\hline PPBS (mg/dl) & 8.03 & 0.001 & 63.43 & 0.000 & 19.42 & 0.000 & 62.57 & 0.000 \\
\hline $\mathrm{HbA}_{1} \mathrm{C}(\%)$ & 26.42 & 0.000 & 137.68 & 0.000 & 37.28 & 0.000 & 143.02 & 0.000 \\
\hline T.Cholesterol (mg/dl) & 2.15 & 0.05 & 2.73 & 0.05 & 3.23 & 0.05 & 2.45 & 0.05 \\
\hline Triglycerides (mg/dl) & 1.93 & 0.05 & 2.87 & 0.05 & 2.43 & 0.05 & 4.65 & 0.01 \\
\hline HDL-C (mg/dl) & 2.24 & 0.05 & 3.07 & 0.05 & 3.14 & 0.05 & 2.41 & 0.05 \\
\hline LDL-C (mg/dl) & 4.78 & 0.05 & 3.74 & 0.05 & 1.91 & 0.05 & 3.04 & 0.05 \\
\hline VLDL-C (mg/dl) & 1.93 & 0.05 & 2.87 & 0.05 & 2.43 & 0.05 & 4.65 & 0.010 \\
\hline SGOT (U/L) & 0.03 & N.S & 4.70 & 0.01 & 1.50 & 0.242 & 4.29 & 0.01 \\
\hline SGPT (U/L) & 0.57 & N.S & 0.56 & N.S & 0.11 & N.S & 0.71 & N.S \\
\hline Alk.Phosphatase (U/L) & 0.07 & N.S & 16.90 & 0.000 & 4.06 & 0.05 & 12.29 & 0.000 \\
\hline Total Protein (g/dl) & 1.97 & 0.05 & 0.14 & N.S & 1.94 & 0.05 & 0.71 & N.S \\
\hline Albumin (g/dl) & 1.00 & N.S & 0.93 & N.S & 1.40 & N.S & 0.80 & N.S \\
\hline
\end{tabular}

\section{DiSCUSSION}

Type $2 \mathrm{DM}$ epidemic has been imputed to urbanization and environmental transition promoting sedentary behaviour and overnutrition. In 2017, global urban diabetic population was 279.2 million and is expected to increase to 628.6 million by 2045 chiefly due to globalisation [10]. In 2019, over 30 million people have been diagnosed with diabetes in India.

Type 2 Diabetes Mellitus is more common in males rather than females while the risk for developing CVD and other complications are higher (25-50\%) in women compared to men [6, 30]. As per Government survey of India (2015-2019) prevalence of Type 2 DM in males was $12 \%$ and $11.7 \%$ in females [11]. In our result we recorded $40.0 \%$ males and $60 \%$ females with UnComplicated Type 2 Diabetes. Complicated Type 2 diabetes was recorded in $53.0 \%$ males and $47.0 \%$ females (Table 2).

Duration of Diabetes is the strongest predictor for the developement and progression of vascular complications of the disease. Poor glycemic Control in Indian diabetic population alongwith lack of timely screening predisposes the patients to severe vascular diabetic complications, sometimes even at the time of diagnosis of disease. In our study we observed $34.0 \%$ UnComplicated Type 2 Cases and $13.0 \%$ Complicated Type 2 Cases in $0-1$ years duration of diabetes. $49.0 \%$ and $35.0 \%$ Cases (both groups respectively) were observed in $>1-5$ years group, $12.0 \% \& 36.0 \%$ Cases (both groups respectively) in $>5$-10years group and $5.0 \%$ and $26.0 \%$ Cases in $>10+$ years group (Table 5).

Smokers Vs Nonsmokers Control subjects reflected significantly low values of Vitamin D $(\mathrm{P}<0.05)$ and high TG, VLDL-C $(\mathrm{P}<0.01)$ in the
Smokers. Cigarette smoke decreases the production of the active form of Vitamin D and also affects the expression levels of the vitamin in lung epithelial cells. This effect intensifies with higher pack years of smoking and causes chronic inflammation and deterioration of lung functions. Additionally smoking is a well-known risk factor for arteriosclerosis and diabetes mellitus. Many studies have reported high TG, LDL-C, VLDL-C and Low HDL-C concentrations in smokers $[12,13]$ similar to our study results.

Alcoholic Vs Non-Alcoholic comparison reflected significantly high value of Alkaline Phosphatase $(\mathrm{P}<0.05)$ by the former group. Exposure to excessive ethanol adversely affects the metabolism of Vitamin $\mathrm{D}$ by depleting enzymes involved in converting $25(\mathrm{OH}) \mathrm{D}_{3}$ to $1,25(\mathrm{OH})_{2} \mathrm{D}_{3}$ thus reducing Vitamin $\mathrm{D}$ levels as observed in our study [14] A.V Skalny et al., [15] demonstrated that use of alcohol induces modulation of Zinc transporters resulting in decreased Zinc levels in lungs, liver, gut and brain. Zinc deficiency in different organs causes systemic inflammation, endotoxemia, alcoholic liver disease and accumulation of neutroxic metabolites. Alcoholism is usually accompanied by mild increase in liver enzymes usually indicative of Alcoholic Liver Disease. In our study we found slight increase in Alkaline Phosphatase in Alcoholics when compared to Non-Alcoholic Control subjects. No other Parameter was found to be significant in this comparison.

\section{Conclusion}

Our study clearly indicates significant decline in Vitamin D, Calcium and Phosphorus levels in Type 2 Diabetes and its associated complications. Faulty lifestyle, altered food habits \& diminished physical activity culpable for obesity aggravates the pre-existing 
insulin resistance state. Therefore timely assessing of Vitamin D and various minerals at the start and even before the onset of diabetes will be certainly supportive in diabetes management. Towering figure of this epidemic demands varied biochemical approach along with conventional glucose monitoring goals. Hence, our study strongly advocates the regular assessment of Vitamin D and Minerals for beforehand diagnosis of the diabetes and its vascular adversities.

\section{Authorship}

All Authors have done Equal contribution for research work.

\section{ACKNOWLEDGEMENT}

We would like to acknowledge the whole Biochemistry department staff as well as lab technicians.

\section{REFRENCES}

1. Katharina Walti: Magnesium Deficiency in Type 2 Diabetes. Diss ETH No. 15168, Swiss Federal Institute of Technology Zurich, Switzerland; 2003.

2. Sergeev IN. Calcium as a Mediator of 1,25dihydroxy vitamin D3 - induced apoptosis. J steroid Biochem Mol Biol.2004; 89-90; 419-425.

3. Sergeev N,Rhoten WB. 1, 25-dihydroxy Vitamin D 3 evokes oscillations of intracellular calcium in a pancreatic Beta-cell line. Endocrinology. 1995;136(7):2852-2861.

4. Pittas AG, Bess DH. Vitamin D and Diabetes. J steroid Biochem Mol Biol. 2010;121(1-2);425-429.

5. Zemel MB. Nutritional and endocrine modulation of intracellular calcium: implications in obesity insulin resistance and hypertension Mol cell Biochem. 1999; 188:129-136.
6. Reusch JE, Begum N, Sussman KE, Draznin B. Regulation of GLUT-4 phosphorylation by intracellular calcium in adipocytes. Endocrinology. 1991; 129:3269-3273.

7. Zemel MB, Shi H, Greer B, Divrienzo D, Zemel PC. Regulation of adioposity by dietary calcium.FASEBJ. 2000; 14:1132-1138

8. MC Carty MF, Thomas CA. PTH excess may provide weight gain by impending catecholamine induced lipolysis-implications for the impact of calcium, Vitamin D and Alcohol on body weight. Med Hypothesis.2003; 61:535-542

9. Food and Nutrient Boared Institute of Medicine. Dietary refrences intakes for calcium, phosphorus, magnesium, Vitamin D and flouride. Washington DC: National Academey Press; 2003.

10. Vijay Vishwanathan.Diabetes voice online.2017; 64 (4):26-29.

11. Shimona Kanwar.Type- 2 diabetes: $46 \mathrm{~L}$ of patients in India are under 40.Times of India; 2016.

12. Haglin LM, Tornkvist B, Backman LO. High serum Phosphate and triglyceride levels in smoking women and men with CVD risk and type 2 diabetes. Diabetol Metab Synder. 2014;6(1):39.

13. Craig WY, Palomaki GE, Haddow JE. Cigarette smoking and serum lipid and lipoprotein concentrations: an analysis of published data.BMJ: 298(6676):784-8.

14. Ogunsakin O, Hottor T, Mehta A, Lichtveld M, McCaskill M. Chronic ethanol exposure effects on vitamin D levels among subjects with alcohol use disorder. Environmental health insights. 2016 Jan; 10:EHI-S40335.

15. Skalny AV, Skalnaya MG, Grabeklis AR, Skalnaya AA, Tinkov AA. Zinc deficiency as a mediator of toxic effects of alcohol abuse. European journal of nutrition. 2018 Oct 1;57(7):2313-22. 